\title{
NEUTRINOS FROM WIMP ANNIHILATIONS
}

\author{
TOMMY OHLSSON \\ Department of Theoretical Physics, School of Engineering Sciences, Royal Institute of \\ Technology (KTH) - AlbaNova University Center, Roslagstullsbacken 21, SE-106 91 \\ Stockholm, Sweden \\ E-mail: tommy@theophys.kth.se
}

Royal Swedish Academy of Sciences (KVA), P.O. Box 50005, SE-10405 Stockholm, Sweden

\begin{abstract}
In this talk, we make an improved analysis on the production of neutrinos coming from WIMP annihilations inside the Sun as well as the Earth. We treat both neutrino interaction and oscillation effects in a consistent threeflavor framework. Our numerical simulations are performed in an event-based setting, which is useful for both theoretical studies and creating neutrino telescope Monte Carlo simulations. We find that the yield of muon-type neutrinos is enhanced or suppressed depending on the dominant WIMP annihilation channel. In addition, we find that oscillations can significantly affect the neutrino yields from WIMP annihilations in the Sun. Effectively, the neutrino flavors are mixed. Finally, for the Earth, the oscillations have no large impact at energies for the new neutrino telescopes such as IceCube, ANTARES, and NESTOR.
\end{abstract}

Keywords: WIMP annihilations; neutrinos; neutrino interactions; neutrino oscillations

\section{Introduction}

Cosmological and astronomical observations show that the energy and matter in the Universe consist of $4 \%$ ordinary baryonic matter, $23 \%$ dark matter, and $73 \%$ dark energy. ${ }^{1}$ One of the most plausible dark matter candidates are so-called weakly interacting massive particles (WIMPs). In particular, neutralinos are promising such WIMP candidates.

WIMPs in the Milky Way halo can scatter in the Sun/Earth and be gravitationally bound to the Sun/Earth. ${ }^{2-6}$ Eventually, they will scatter again and sink to the core. In the core, WIMPs will accumulate and can annihilate and produce neutrinos. 


\section{Simulations}

We simulate 13 different annihilation channels using Pythia $6.400 .^{7}$ The annihilation channels are

$$
\chi \chi \rightarrow u \bar{u}, d \bar{d}, s \bar{s}, c \bar{c}, b \bar{b}, t \bar{t}, \tau^{+} \tau^{-}, W^{+} W^{-}, Z^{0} Z^{0}, g \bar{g}, \nu_{e} \bar{\nu}_{e}, \nu_{\mu} \bar{\nu}_{\mu}, \nu_{\tau} \bar{\nu}_{\tau}
$$

and the simulations are performed with a full three-flavor Monte Carlo code including both neutrino interactions and neutrino oscillations. The code is event-based, and thus, useful for both theoretical analysis and building a neutrino telescope Monte Carlo. We perform simulations for the following 19 WIMP masses: 10, 25, 50, 80.3, 91.2, 100, 150, 176, 200, 250, 350, 500, $750,1000,1500,2000,3000,5000$, and $10000 \mathrm{GeV}$. For each mass and annihilation channel, we perform $2.5 \cdot 10^{6}$ annihilations. In addition, we perform the simulations for seven different sets of neutrino oscillation parameters. We collect all neutrinos $\left(\nu_{e}, \nu_{\mu}, \nu_{\tau}\right)$ and antineutrinos $\left(\bar{\nu}_{e}, \bar{\nu}_{\mu}, \bar{\nu}_{\tau}\right)$, keeping track of the flavors separately.

\section{Neutrino interactions and neutrino oscillations}

\subsection{Neutrino interactions}

On the way out of the Sun, neutrinos can participate in both chargedand neutral-current interactions. Neutral currents degrade the energy of the neutrinos, whereas charged currents give charged leptons: electrons and muons are stopped before they can give neutrinos, but taus will decay and give neutrinos (regeneration). Simulations are performed using CTEQ6 DIS parton distribution functions. ${ }^{8}$

\subsection{Neutrino oscillations}

A completely general three-flavor neutrino oscillation framework (with matter effects included) as well as the realistic Standard Solar Model ${ }^{9}$ (for propagation in the Sun) and the Preliminary Reference Earth Model ${ }^{10}$ (for propagation in the Earth) are used, i.e., we calculate the total evolution operator for neutrino propagation using accurate matter density profiles for both the Sun and the Earth. In matter, we decompose the total evolution operator $S$ in $k$ factors as

$$
S=S_{k} S_{k-1} \ldots S_{2} S_{1},
$$

where $S_{i}$ is the evolution operator in layer $i$ with constant matter density. ${ }^{11}$ The total evolution operator contains the information of evolution for the 
neutrinos and includes of course both amplitudes and phases. The probability of neutrino flavor transition after propagation through matter is then given by

$$
P_{\alpha \beta}=\left|S_{\beta \alpha}\right|^{2} .
$$

At the surface of the Sun, at the distance of $1 \mathrm{AU}$, and at an actual detector on Earth, we obtain the neutrino yields in a general format (with amplitudes and phases). The neutrino oscillation parameters used are: $\theta_{12}=33.2^{\circ}$, $\theta_{13}=0,5^{\circ}, 10^{\circ}, \theta_{23}=45.0^{\circ}, \delta=0, \Delta m_{21}^{2}=8.1 \cdot 10^{-5} \mathrm{eV}^{2}$, and $\Delta m_{31}^{2}=$ $\pm 2.2 \cdot 10^{-3} \mathrm{eV}^{2}$ (normal and inverted mass hierarchies).

\section{Neutrino production}

Although we have preformed simulations and computed spectra of neutrino production for a number of annihilation channels, WIMP masses, as well as for seven sets of neutrino oscillation parameters, we present only results for the annihilation of $250 \mathrm{GeV}$ WIMPs into $\tau^{+} \tau^{-}$, using the following set of values for the neutrino oscillation parameters (std. osc.) $\theta_{12}=33.2^{\circ}, \theta_{13}=$ $0, \theta_{23}=45.0^{\circ}, \delta=0, \Delta m_{21}^{2}=8.1 \cdot 10^{-5} \mathrm{eV}^{2}$, and $\Delta m_{31}^{2}=2.2 \cdot 10^{-3} \mathrm{eV}^{2}$ as well as no oscillations, in this talk. Nevertheless, the other spectra are publicly available, see Ref. ${ }^{12}$

In Figs. 1 and 2, we show the neutrino yields at production in the center of the Sun and at the surface of the Sun (using matter neutrino oscillations in the Sun for the propagation from the center of the Sun to the surface of the Sun), respectively. From these two figures, two main effects can be observed:

(1) Oscillations effectively mix muon and tau neutrinos, whereas the electron neutrinos remain essentially unmixed.

(2) There is a pile-up of events at low energies.

The first effect is due to the electron neutrino being equivalent to one of the matter eigenstates when propagating in matter with large matter density, whereas the second effect comes from charged- and neutral-current interactions.

Next, we propagate the neutrinos from the Sun to the Earth, using vacuum neutrino oscillations. In Fig. 3, we show the neutrino yields at the distance of $1 \mathrm{AU}$, i.e., the distance between the Sun and the Earth. Again, two main effects can be observed:

(1) Electron neutrinos are effectively mixed with muon and tau neutrinos solely due to vacuum oscillations governed by the small mass squared difference $\Delta m_{21}^{2}$. 


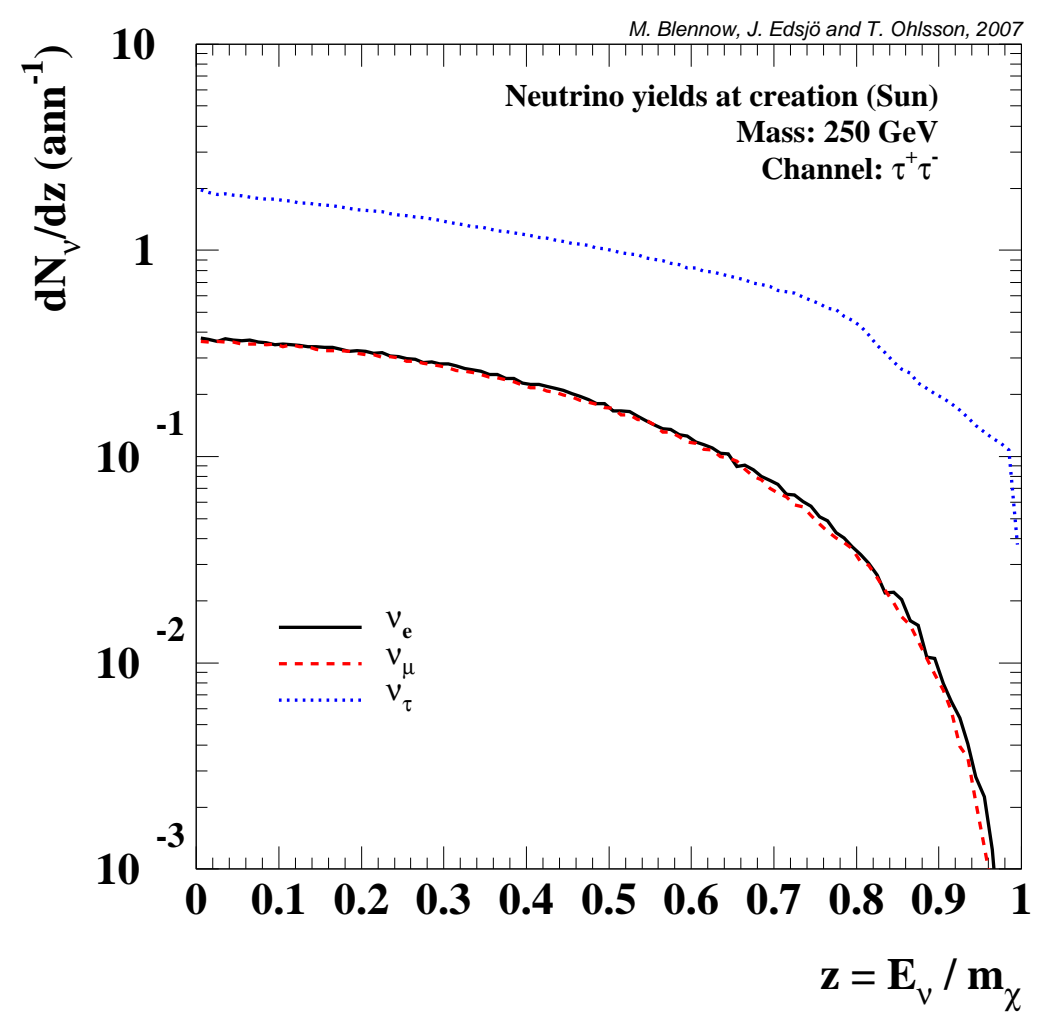

Fig. 1. Neutrino yields at creation in the center of the Sun. The figure has been adopted from Ref. ${ }^{13}$

(2) The yields of muon and tau neutrinos essentially coincide due to fast oscillations governed by the large mass squared difference $\Delta m_{31}^{2}$ and the leptonic mixing angle $\theta_{23}$.

Then, we propagate the neutrinos through the Earth to an actual detector, using matter neutrino oscillations in the Earth. The simulations are performed with a time stamp to include effects of the Earth's distance to the Sun (due to the eccentricity of the Earth's orbit around the Sun) as well as rotation (which affects the distance traversed through the Earth). In Fig. 4, we present the neutrino yields at a fictive detector at latitude $-90^{\circ}$, which could e.g. be the IceCube neutrino telescope, and we have averaged the result of the simulations over half a year. In this case, the 


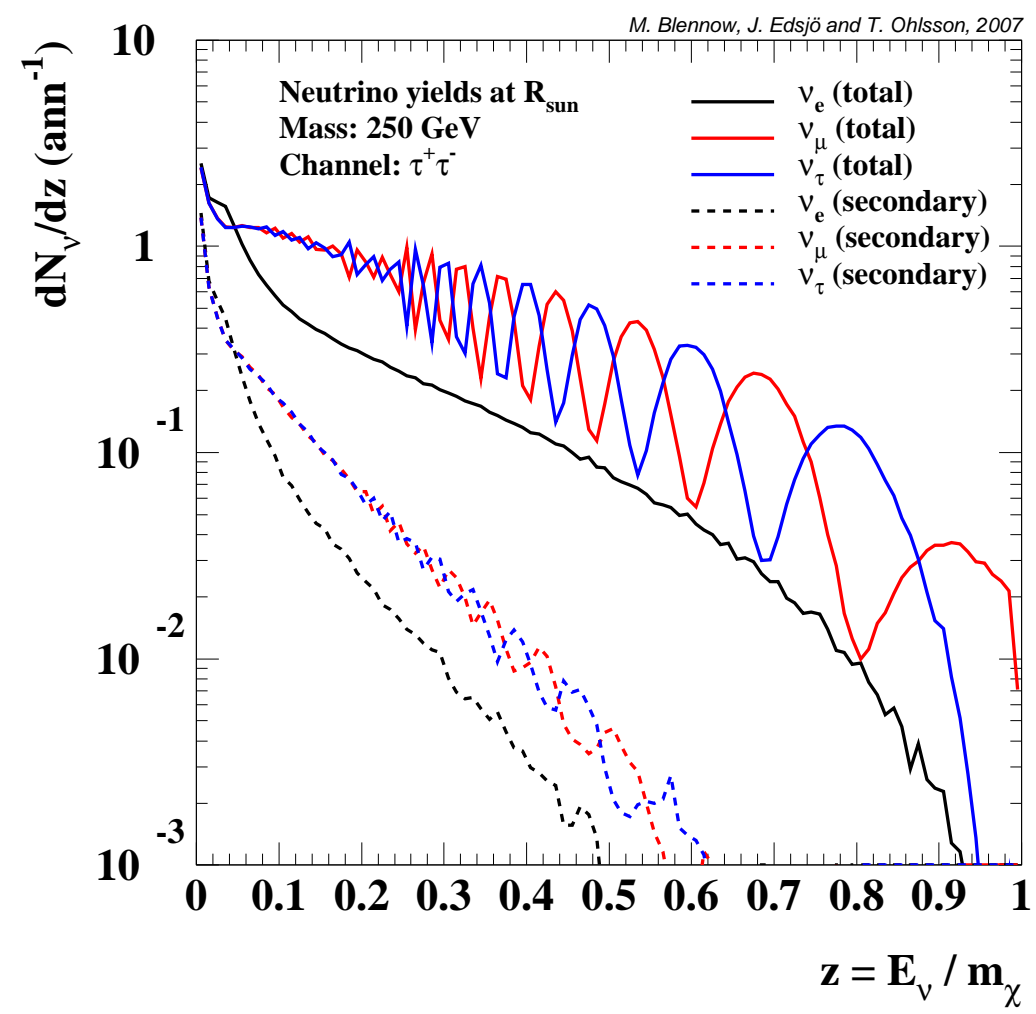

Fig. 2. Neutrino yields at the surface of the Sun. The secondary neutrinos are coming from $\tau^{ \pm}$decays after $\nu_{\tau} / \bar{\nu}_{\tau}$ charged-current interactions. The figure has been adopted from Ref. ${ }^{13}$

main effect is that the oscillations that were present before are now almost completely washed out. In fact, matter effects from the passage through the Earth are negligible and the main reason for this "averaging" comes from the eccentricity of the Earth's orbit.

Finally, we consider production of neutrinos from WIMP annihilations in the center of the Earth. In Fig. 5, we present the neutrino yields at a possible detector. The effects of oscillations are seen as a decrease of the tau neutrino yield and an increase of the muon neutrino yield at low energies (below about $50 \mathrm{GeV}$ ). For higher energies, that are of most interest for the currently planned neutrino telescopes such as IceCube, ANTARES, and 


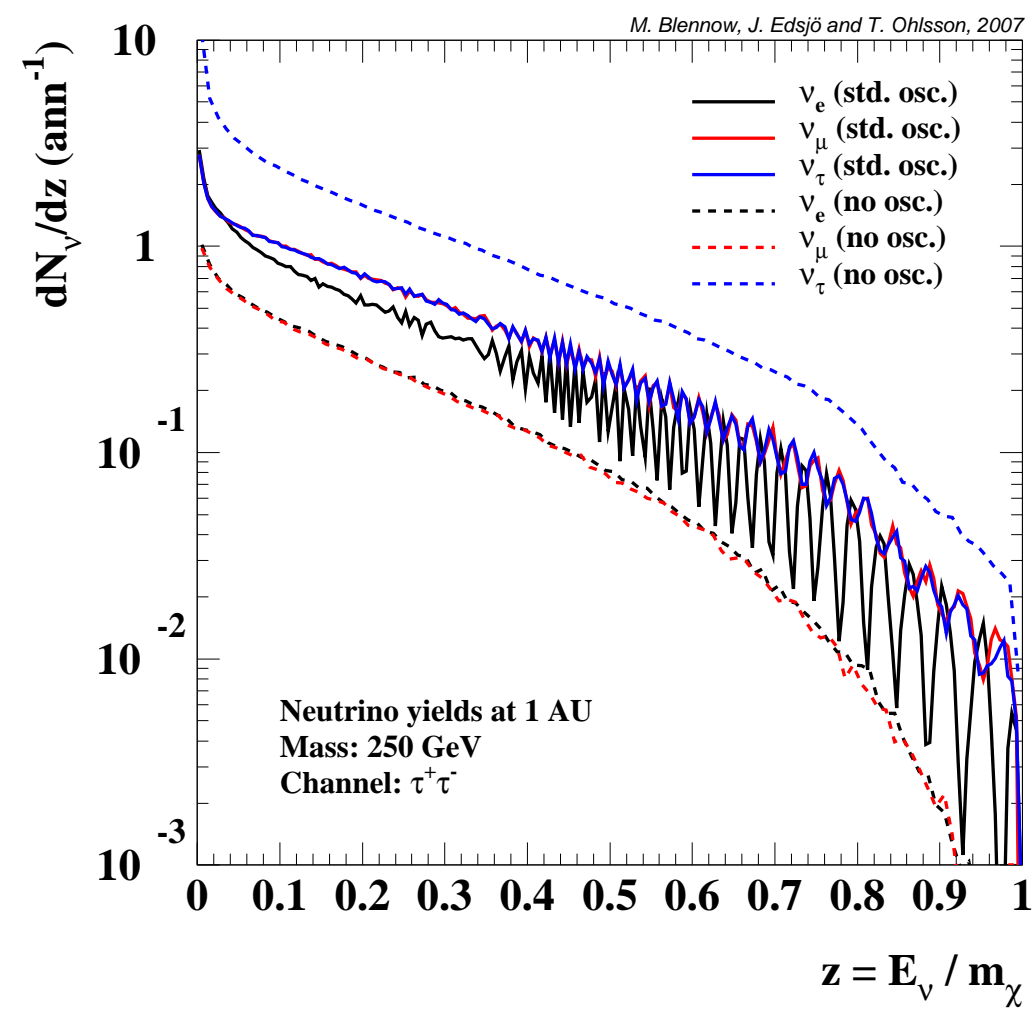

Fig. 3. Neutrino yields at the distance of $1 \mathrm{AU}$ for both with and without neutrino oscillations. The figure has been adopted from Ref. ${ }^{13}$

NESTOR, neutrino oscillations do not have a large effect on the neutrino yields from annihilations of WIMPs inside the Earth.

\section{Conclusions}

For WIMP annihilations in the center of the Sun, using typical WIMP masses [i.e., $\left.\left(10-10^{5}\right) \mathrm{GeV}\right]$, neutrino oscillations effectively average muon and tau neutrinos on the way out of the Sun, average electron and muon/tau neutrinos on the way through the Earth, and wash out the remaining oscillation pattern in the spectra due to the eccentricity of the Earth's orbit around the Sun. 


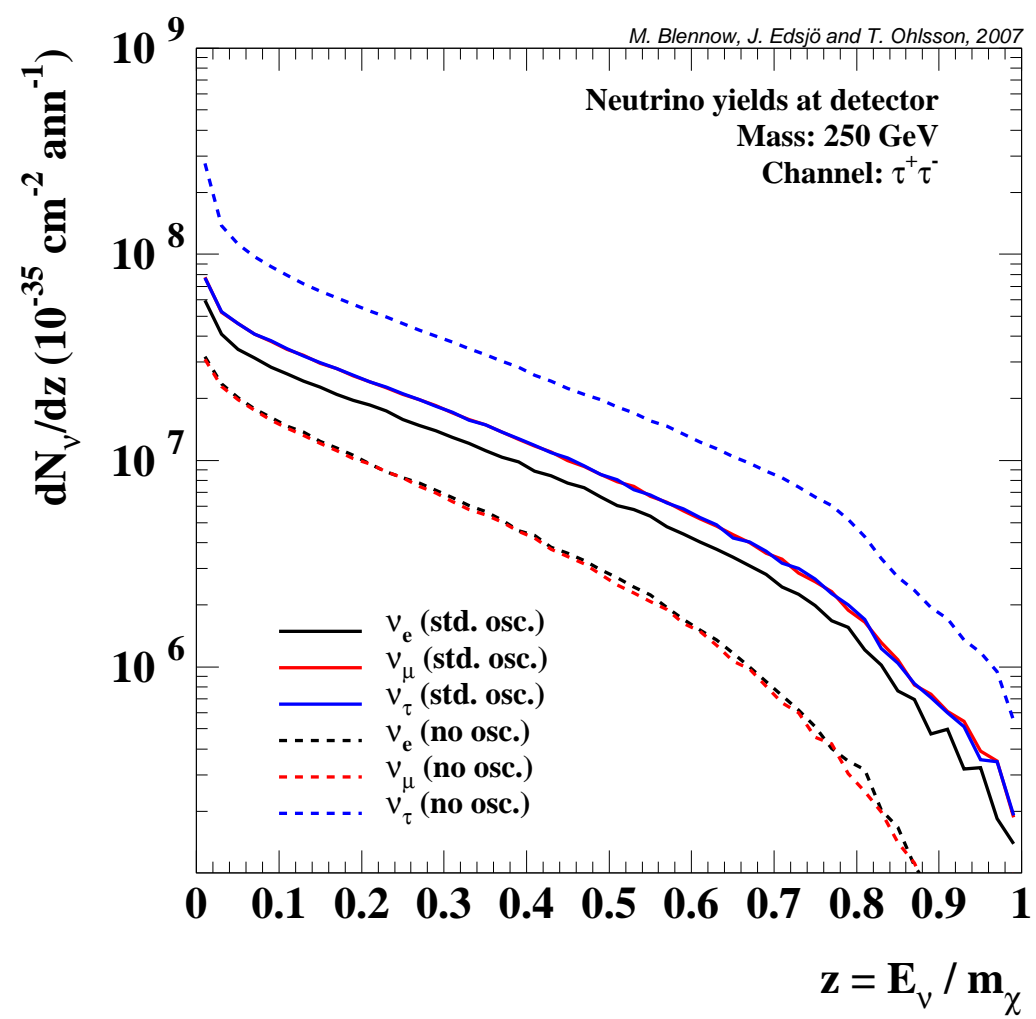

Fig. 4. Time-averaged neutrino yields at a detector at latitude $-90^{\circ}$ for both with and without neutrino oscillations. The figure has been adopted from Ref. ${ }^{13}$

For WIMP annihilations in the center of the Earth, there are essentially no effects of neutrino oscillations (except below $50 \mathrm{GeV}$ where muon and tau neutrinos are mixed).

\section{Acknowledgments}

I would like to thank Mattias Blennow and Joakim Edsjö for useful collaboration that led to the publication ${ }^{13}$ upon which this talk is based. In addition, I would like to thank the organizers of Dark 2007 for giving me the opportunity to give this talk.

This work was supported by the Royal Swedish Academy of Sciences 


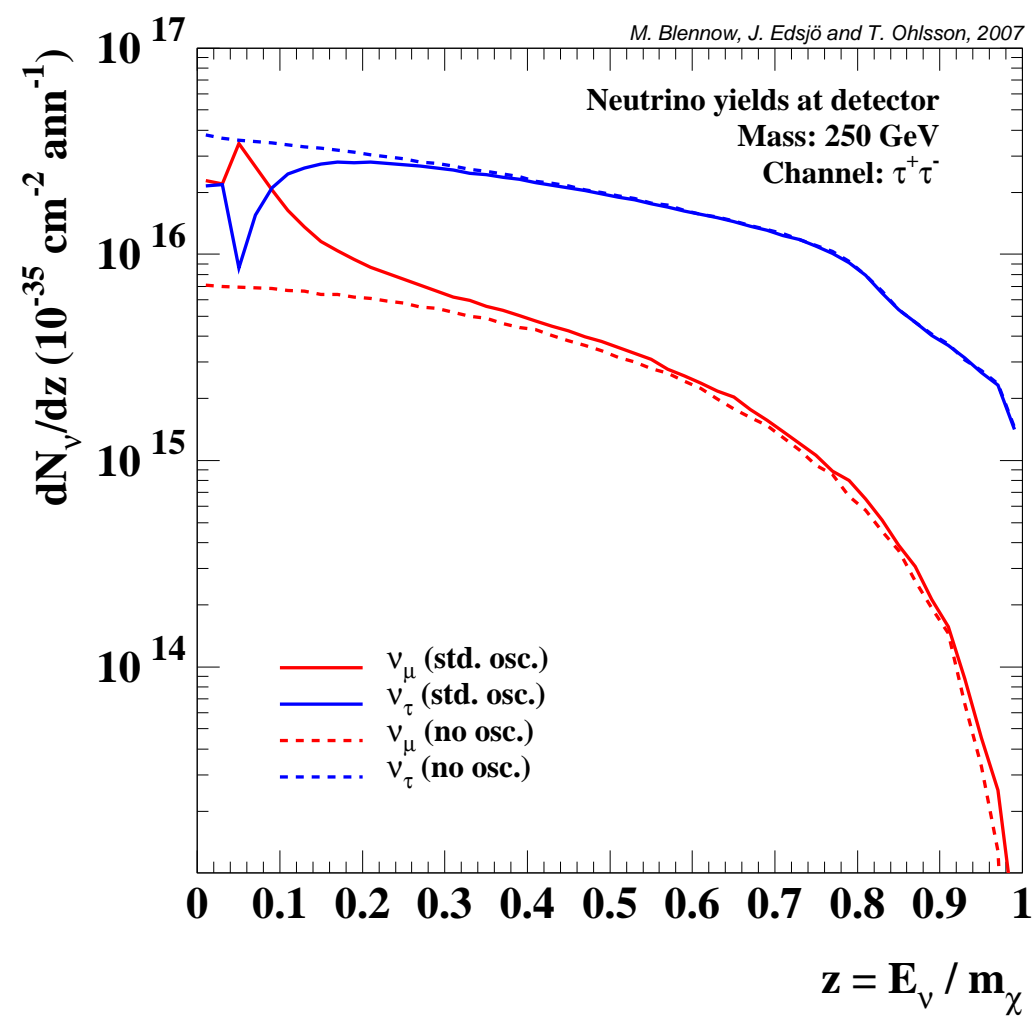

Fig. 5. Neutrino yields (from WIMP annihilations in the center of the Earth) at a detector at the surface of the Earth for both with and without neutrino oscillations. The electron neutrino yields are not plotted, since they are identical to the muon neutrino yields without neutrino oscillations. The figure has been adopted from Ref. ${ }^{13}$

(KVA) and the Swedish Research Council (Vetenskapsrådet), Contract No. 621-2005-3588.

\section{References}

1. D. N. Spergel et al. [WMAP Collaboration], Astrophys. J. Suppl. 170, 377 (2007) [arXiv:astro-ph/0603449].

2. W. H. Press and D. N. Spergel, Astrophys. J. 296, 679 (1985).

3. K. Freese, Phys. Lett. B 167, 295 (1986).

4. L. M. Krauss, M. Srednicki, and F. Wilczek, Phys. Rev. D 33, 2079 (1986).

5. T. K. Gaisser, G. Steigman, and S. Tilav, Phys. Rev. D 34, 2206 (1986). 
6. J. Lundberg and J. Edsjö, Phys. Rev. D 69, 123505 (2004) [arXiv:astro$\mathrm{ph} / 0401113]$.

7. T. Sjöstrand, S. Mrenna, and P. Skands, JHEP 0605, 026 (2006) [arXiv:hep$\mathrm{ph} / 0603175]$.

8. J. Pumplin, D. R. Stump, J. Huston, H. L. Lai, P. Nadolsky, and W. K. Tung, JHEP 0207, 012 (2002) [arXiv:hep-ph/0201195].

9. J. N. Bahcall, A. M. Serenelli, and S. Basu, Astrophys. J. 621, L85 (2005) [arXiv:astro-ph/0412440].

10. A. M. Dziewonski and D. L. Anderson, Phys. Earth Planet. Interiors 25, 297 (1981).

11. T. Ohlsson and H. Snellman, Phys. Lett. B 474, 153 (2000) [arXiv:hep$\mathrm{ph} / 9912295]$.

12. Our results are publicly available at www.physto.se/ ${ }^{\sim e d s j o / w i m p s i m ~}$

13. M. Blennow, J. Edsjö, and T. Ohlsson, arXiv:0709.3898 [hep-ph]. 